\title{
Irregularity Measures of Graph
}

\section{E. Milovanović, E. Glogić, I. Milovanović, M. Cvjetković}

\begin{abstract}
Let $G=(V, E), V=\{1,2, \ldots, n\}$, be a simple graph without isolated vertices, with vertex degree sequence $d_{1} \geq d_{2} \geq \cdots \geq d_{n}>0, d_{i}=d(i)$. A graph $G$ is regular if and only if $d_{1}=d_{2}=\cdots=d_{n}$. A graph invariant $I(G)$ is measure of irregularity of graph $G$ with the property $I(G)=0$ if and only if $G$ is regular, and $I(G)>0$ otherwise. In this paper we introduce some new irregularity measures.
\end{abstract}

Keywords: Irregularity measures (of graph), Zagreb index, Randić index

\section{Introduction and preliminaries}

Let $G=(V, E), V=\{1,2, \ldots, n\}, E=\left\{e_{1}, e_{2}, \ldots, e_{m}\right\}$,be a simple graph without isolated vertices. Denote by $d_{i}=d(i), i=1,2, \ldots, n$ vertex degree and by $d\left(e_{i}\right), i=1,2, \ldots, m$ an edge degree, whereby for each edge $e=\{i, j\} \in E$ holds $d(e)=d_{i}+d_{j}-2$. Without affecting the generality we can assume that $d_{1} \geq d_{2} \geq \cdots \geq d_{n}>0$ and $d\left(e_{1}\right) \geq d\left(e_{2}\right) \geq$ $\cdots \geq d\left(e_{m}\right) \geq 0$. By $i \sim j$ we denote an edge connecting vertices $i$ and $j$, and by $e_{i} \sim e_{j}$ that edges $e_{i}$ and $e_{j}$ are adjacent.

In the text that follows we shall list some previously defined degree-based graph invariants needed for our work. For the details of degree-based topological indices one can refer to $[17,18,25,37,38]$.

The first and the second Zagreb indices, $M_{1}$ and $M_{2}$, are, respectively, defined as [20]

$$
M_{1}(G)=M_{1}=\sum_{i=1}^{n} d_{i}^{2}, \quad \text { and } \quad M_{2}(G)=M_{2}=\sum_{i \sim j} d_{i} d_{j} .
$$

Reformulated first and second Zagreb indices, $E M_{1}$ and $E M_{2}$, are defined as [29, 41]

$$
E M_{1}(G)=E M_{1}=\sum_{i=1}^{m} d\left(e_{i}\right)^{2} \quad \text { and } \quad E M_{2}(G)=E M_{2}=\sum_{e \sim f} d(e) d(f) .
$$

Manuscript received December 21, 2014; accepted April 4, 2015.

E. Milovanović, I. Milovanović are with the Faculty of Electronic Engineering, A. Medvedeva 14, 18000 Niš, Serbia; E. Glogić is with the State University of Novi Pazar, 36300 Novi Pazar, Serbia; M. Cvjetković is with the Business School of Applied Studies, 18420 Blace, Serbia. 
Modified first and second Zagreb indices, ${ }^{m} M_{1}$ and ${ }^{m} M_{2}$, are, respectively, defined by $[33,41]$

$$
{ }^{m} M_{1}(G)={ }^{m} M_{1}=\sum_{i=1}^{n} \frac{1}{d_{i}^{2}} \quad \text { and } \quad{ }^{m} M_{2}(G)={ }^{m} M_{2}=\sum_{i \sim j} \frac{1}{d_{i} d_{j}} .
$$

Finally, reformulated-modified first and second Zagreb indices, ${ }^{m} E M_{1}$ and ${ }^{m} E M_{2}$, are defined as [28]

$$
{ }^{m} E M_{1}(G)={ }^{m} E M_{1}=\sum_{i=1}^{m} \frac{1}{d\left(e_{i}\right)^{2}} \quad \text { and } \quad{ }^{m} E M_{2}(G)={ }^{m} E M_{2}=\sum_{e \sim f} \frac{1}{d(e) d(f)} .
$$

Each graph $G=(V, E)$ has a corresponding line graph $\mathscr{L}=\mathscr{L}(G)$ that is defined by the set of vertices $E$, wherein two vertices from set $E$ are adjacent in graph $\mathscr{L}$ if and only if the corresponding edges are adjacent in the underlying graph $G$. If $G$ has $n$ vertices and $m$ edges, the corresponding line graph has $m$ vertices and $\frac{1}{2}\left(M_{1}-2 m\right)$ edges, whereby

$$
\sum_{i=1}^{n} d_{i}=2 m \quad \text { and } \quad \sum_{i=1}^{m} d\left(e_{i}\right)=M_{1}-2 m
$$

Some well known properties of the above mentioned graph invariants are

$$
\begin{aligned}
& E M_{1}(G)=M_{1}(\mathscr{L}(G)), \quad E M_{2}(G)=M_{2}(\mathscr{L}(G)) \\
& { }^{m} E M_{1}(G)={ }^{m} M_{1}(\mathscr{L}(G)), \quad{ }^{m} E M_{2}(G)={ }^{m} M_{2}(\mathscr{L}(G))
\end{aligned}
$$

"General Randić index", or connectivity index [17, 25] is defined as

$$
R_{\alpha}(G)=R_{\alpha}=\sum_{i \sim j}\left(d_{i} d_{j}\right)^{\alpha}
$$

where $\alpha$ is a real number. It is not difficult to see that for $\alpha=-1, \alpha=0$ and $\alpha=1$, the following is obtained $R_{-1}={ }^{m} M_{2}=\sum_{i \sim j} \frac{1}{d_{i} d_{j}}$ (what is called general Randić index, without quotes) [5,6], $R_{0}=m$ and $R_{1}=M_{2}$, respectively. We are interested in the case when $\alpha=-\frac{1}{2}$ and $\alpha=\frac{1}{2}$. For $\alpha=-\frac{1}{2}$ ordinary Randić index is obtained [34], whereas for $\alpha=\frac{1}{2}$ the reciprocal Randić index is obtained $[14,26]$, i.e.

$$
R_{-1 / 2}(G)=R_{-1 / 2}=\sum_{i \sim j} \frac{1}{\sqrt{d_{i} d_{j}}} \quad \text { and } \quad R_{1 / 2}(G)=R_{1 / 2}=\sum_{i \sim j} \sqrt{d_{i} d_{j}}
$$

In [26] graph invariant ${ }^{m} A$ was introduced and defined as

$$
{ }^{m} A(G)={ }^{m} A=\sum_{i=1}^{n} \frac{1}{d_{i}}
$$


A corresponding edge-invariant ${ }^{m} E A$ is of the form

$$
{ }^{m} E A(G)={ }^{m} E A=\sum_{i=1}^{m} \frac{1}{d\left(e_{i}\right)} .
$$

In the text that follows we mention some results from spectral graph theory needed for our work.

Let $\mathbf{A}$ be the adjacency matrix of graph $G$. Eigenvalues of $\mathbf{A}, \lambda_{1} \geq \lambda_{2} \cdots \geq \lambda_{n}$, are ordinary eigenvalues of graph $G$. Some well known properties of graph eigenvalues are [4]:

$$
\sum_{i=1}^{n} \lambda_{i}=0 \quad \text { and } \quad \sum_{i=1}^{n} \lambda_{i}^{2}=2 m
$$

Denote by $\mathbf{D}=\operatorname{diag}\left\{d_{1}, d_{2}, \ldots, d_{n}\right\}$ a diagonal matrix, where $d_{i}, i=1,2, \ldots, n$ are vertex degrees of $G$. Then $\mathbf{L}=\mathbf{D}-\mathbf{A}$ is the Laplacian matrix of the $G$. Eigenvalues of $\mathbf{L}, \mu_{1} \geq$ $\mu_{2} \geq \cdots \geq \mu_{n-1} \geq \mu_{n}=0$ are Laplacian eigenvalues of the graph $G$. Well known properties of these eigenvalues are [7]

$$
\sum_{i=1}^{n-1} \mu_{i}=\sum_{i=1}^{n} d_{i}=2 m \quad \text { and } \quad \sum_{i=1}^{n-1} \mu_{i}^{2}=\sum_{i=1}^{n} d_{i}^{2}+\sum_{i=1}^{n} d_{i}=M_{1}+2 m .
$$

Because it is assumed that graph $G$ has no isolated vertices, the matrix $\mathbf{D}$ is nonsingular and therefore matrix $\mathbf{D}^{-1 / 2}$ exits. $\mathbf{L}^{*}=\mathbf{D}^{-1 / 2} \mathbf{L D}^{-1 / 2}=\mathbf{I}-\mathbf{D}^{-1 / 2} \mathbf{A} \mathbf{D}^{-1 / 2}$ is called normalized Laplacian matrix of the graph $G$. Eigenvalues of $\mathbf{L}^{*}, \rho_{1} \geq \rho_{2} \geq \cdots \geq \rho_{n}=0$, are normalized Laplacian eigenvalues of graph $G$. Well known properties of these eigenvalues are [44]

$$
\sum_{i=1}^{n-1} \rho_{i}=n \quad \text { and } \quad \sum_{i=1}^{n-1} \rho_{i}^{2}=n+2 R_{-1}
$$

A graph $G$ is regular if and only if $d_{1}=d_{2}=\cdots=d_{n}$, otherwise it is irregular. A graph invariant $I(G)$ is measure of irregularity of graph $G$ with the property $I(G)=0$ if and only if $G$ is regular, and $I(G)>0$ otherwise.With $\operatorname{LIrr}(G)$ we will denote irregularity measure of the line graph $\mathscr{L}(G)$ of the underlying graph $G$. Note that if graph $G$ is regular than $\mathscr{L}(G)$ is regular as well, but the reverse is not necessarily valid.

At the end of this introductory section we recall Chebyshev inequality (see [30, 31], given in the following theorem, which is necessary for our work.

Theorem 1 Let $p_{1}, p_{2}, \ldots, p_{n}$ be positive real numbers and $a_{1}, a_{2}, \ldots, a_{n}$ and $b_{1}, b_{2}, \ldots, b_{n}$ real sequences of the same monotonicity. Then

$$
\sum_{i=1}^{n} p_{i} \sum_{i=1}^{n} p_{i} a_{i} b_{i} \geq \sum_{i=1}^{n} p_{i} a_{i} \sum_{i=1}^{n} p_{i} b_{i} .
$$

Equality holds if and only if $a_{1}=a_{2}=\cdots=a_{n}$ or $b_{1}=b_{2}=\cdots=b_{n}$.

If $a_{1}, a_{2}, \ldots, a_{n}$ and $b_{1}, b_{2}, \ldots, b_{n}$ are real sequences of opposite monotonicity than the opposite inequality holds in (1). 


\section{Some old and some new irregularity measures}

In this section we mention some known and introduce some new irregularity measures.

Albertson [2] introduce the irregularity measure, nowadays called Albertson measure, as

$$
\operatorname{Irr}_{A}(G) \sum_{i \sim j}\left|d_{i}-d_{j}\right|
$$

Let us note that Fath-Tabar [13] called this irregularity measure the third Zagreb index.

Inspired by the measure $\operatorname{Irr}_{A}(G)$ Abdo et all [1] introduced irregularity measure referred to as total irregularity, defined as

$$
\operatorname{Irr}_{t}(G)=\frac{1}{2} \sum_{i, j \in V}\left|d_{i}-d_{j}\right| .
$$

In accordance with [19] we will call this measure Abdo-Brandt-Dimitrov irregularity measure.

In [15] Goldberg noticed that the simplest irregularity measure is

$$
d(G)=d_{1}-d_{n} .
$$

We will call this measure as Golberg irregularity measure, and in accordance with [19] consider it in the form

$$
\operatorname{Irr}_{g}(G)=\frac{d_{1}}{d_{n}}-1 .
$$

Nikiforov [32] introduced irregularity measure referred to as degree deviatioan defined as

$$
S(G)=\left|\sum_{i=1}^{n} d_{i}-\frac{2 m}{n}\right|
$$

We will call this measure Nikiforov irregularity measure and denote it by $\operatorname{Ir}_{N}(G)$.

Bell [3] considered the variance of vertex degrees as irregularity measure

$$
\operatorname{VAR}(G)=\frac{1}{n} \sum_{i=1}^{n}\left(d_{i}-\frac{2 m}{n}\right)^{2} .
$$

It is not difficult to see that irregularity measures $S(G)$ and $\operatorname{VAR}(G)$ are special case of the following graph invariant

$$
R_{p}(G)=\left(\frac{1}{n^{p-1}} \sum_{i=1}^{n}\left|d_{i}-\frac{2 m}{n}\right|^{p}\right)^{1 / p}, \quad p \geq 1 .
$$


In [11] Edwards considered the quantity

$$
1+C_{\gamma}^{2}=\frac{n M_{1}}{4 m^{2}}
$$

where $C_{\gamma}$ is non negative real number. Edwards proved that $C_{\gamma}=0$ if and only if $G$ is a regular graph. This means that the following inequality holds

$$
M_{1} \geq \frac{4 m^{2}}{n}
$$

Equality holds if and only if $G$ is a regular graph. More about (4) one can refer to $[9,16$, $23,27,36,42]$. Based on (4) in [19] a measure called Edwards irregularity measure was defined as

$$
\operatorname{Irr}_{E}(G)=\sqrt{\frac{n M_{1}}{4 m^{2}}-1}
$$

Let us note that since

$$
\operatorname{VAR}(G)=\frac{1}{n} \sum_{i=1}^{n}\left(d_{i}-\frac{2 m}{n}\right)^{2}=\frac{n M_{1}-4 m^{2}}{n^{2}}
$$

irregularity measure defined by (2) is not different from the one defined by (5). The same applies to irregularity measure proposed in [21] which is defined as

$$
I R M_{1}(G)=M_{1}-\frac{4 m^{2}}{n}
$$

Note, also, that inequality (4) can be obtained from (1) for $p_{i}=1, a_{i}=b_{i}=d_{i}, i=$ $1,2, \ldots, n$.

For $n:=m$ and $m:=\frac{1}{2}\left(M_{1}-2 m\right)$ from inequality (4) the following is obtained

$$
E M_{1} \geq \frac{\left(M_{1}-2 m\right)^{2}}{m}
$$

with equality holding if and only if $\mathscr{L}(G)$ is a regular graph. The inequality (6) was proved in $[10,23]$. Let us note that inequality (6) can be obtained from (1) for $n:=m, p_{i}=1$, $a_{i}=b_{i}=d\left(e_{i}\right), i=1,2, \ldots, n$.

According to (4) and (6) the following is valid

$$
E M_{1} \geq \frac{4 m(2 m-n)^{2}}{n^{2}}
$$

with equality holding if and only if $G$ is a regular graph. Based on this inequality we can define a new irregularity measure

$$
\operatorname{Irr}_{1}(G)=\sqrt{\frac{n^{2} E M_{1}}{4 m(2 m-n)^{2}}-1}, \quad(2 m \neq n) .
$$


In [10] De proved the following inequality

$$
\left(d_{n}-1\right)^{2} \leq \frac{E M_{1}}{4 m} \leq\left(d_{1}-1\right)^{2}
$$

with equality holding if and only if $G$ is a regular graph. According to the above inequalities we can define two new irregularity measures of De type:

$$
\operatorname{Irr}_{D 1}(G)=\sqrt{1-\frac{E M_{1}}{4 m\left(d_{1}-1\right)^{2}}}, \quad d_{1} \geq 2
$$

and

$$
\operatorname{Irr}_{D 2}(G)=\sqrt{\frac{E M_{1}}{4 m\left(d_{n}-1\right)^{2}}-1}, \quad d_{n} \geq 2 .
$$

Ilić and Stevanović [23] (see also [43]) proved the following inequality

$$
M_{2} \geq \frac{4 m^{3}}{n^{2}}
$$

with equality holding if and only if $G$ is a regular graph. Based on this, a new irregularity measure, Ilić-Stevanović measure, can be defined as

$$
\operatorname{Irr}_{I S}(G)=\sqrt{\frac{n^{2} M_{2}}{4 m^{3}}-1}
$$

In [21] the following irregularity measure was proposed

$$
I R M_{2}(G)=M_{2}-\frac{4 m^{3}}{n^{2}} .
$$

In fact it is nod different from the one defined by (8).

Based on the inequality (7), Ilić and Zhou [24] (see also [10]) proved the following

$$
E M_{2} \geq \frac{\left(M_{1}-2 m\right)^{3}}{2 m^{2}}
$$

with equality holding if and only if $\mathscr{L}(G)$ is a regular graph. By (4) and (9) the following is valid

$$
E M_{2} \geq \frac{4 m(2 m-n)^{3}}{n^{3}}
$$

with equality holding if and only if $G$ is a regular graph. Based on the above we can define a new irregularity measure

$$
\operatorname{Irr}_{2}(G)=\sqrt{\frac{n^{3} E M_{2}}{4 m(2 m-n)^{3}}-1}, \quad 2 m \neq n .
$$


Hao [22] proved the following

$$
{ }^{m} M_{1} \geq \frac{n^{3}}{4 m^{2}}
$$

with equality holding if and only if $G$ is a regular graph.

Let us note that inequality (10) can be obtained from (1). Namely, for $p_{i}=d_{i}, a_{i}=d_{i}=$ $\frac{1}{d_{i}}, i=1,2, \ldots, n$ from (2) we have

$$
\sum_{i=1}^{n} \frac{1}{d_{i}} \geq \frac{n^{2}}{2 m}
$$

For $p_{i}=d_{i}, a_{i}=d_{i}=\frac{1}{d_{i}}, i=1,2, \ldots, n$ inequality (1) becomes

$$
{ }^{m} M_{1} \geq \frac{1}{n}\left(\sum_{i=1}^{n} \frac{1}{d_{i}}\right)^{2} .
$$

From (11) and (12) we obtain (10). Now, having in mind (10) we can define Hao irregularity measure by the following invariant

$$
\operatorname{Irr}_{H}(G)=\frac{4 m^{2 m} M_{1}}{n^{3}}-1 .
$$

Let us note that from inequality (10) directly follows

$$
{ }^{m} E M_{1} \geq \frac{m^{3}}{\left(M_{1}-2 m\right)^{2}}, \quad M_{1} \neq 2 m
$$

with equality holding if and only if $\mathscr{L}(G)$ is a regular graph.

The inequality (11) can be rewritten as

$$
m_{A} \geq \frac{n^{2}}{2 m}
$$

with equality holding if and only if $G$ is a regular graph. According to (14) we can define a new irregularity measure

$$
\operatorname{Irr}_{3}(G)=\frac{2 m^{m} A}{n^{2}}-1 .
$$

Also, note that from (14) directly follows

$$
{ }^{m} E A \geq \frac{m^{2}}{M_{1}-2 m}, \quad M_{1} \neq 2 m
$$

with equality holding if and only if $\mathscr{L}(G)$ is a regular graph. 
Collatz and Sinogowitz [8] have proved that

$$
\lambda_{1} \geq \frac{2 m}{n}
$$

with equality holding if and only if $G$ is a regular graph. Using this inequality in [19] the Collatz-Sinogowitz irregularity measure was defined via

$$
\operatorname{Irr}_{C S}(G)=\frac{n \lambda_{1}}{2 m}-1 .
$$

In the text that follows we mention some irregularity measures defined in terms of "general Randić index"

Elphick and Wocjan [12] considered the edge-variant of Edwards' measure. Based on that in [19] Elphick-Wocjan irregularity measure was defined via

$$
\operatorname{Irr}_{E W}(G)=\sqrt{\frac{n R_{1 / 2}}{2 m^{2}}-1} .
$$

Shi [35] proved that for graphs without isolated vertices the followig inequalities are valid

$$
R_{\alpha} \geq \frac{n}{2} d_{1}^{\alpha} d_{n}^{1+\alpha}, \quad \alpha \in[-1,0]
$$

and

$$
R_{\alpha} \leq \frac{n}{2} d_{n}^{1+2 \alpha}, \quad \alpha \in\left(-\infty,-\frac{1}{2}\right]
$$

with equality holding if and only if $G$ is a regular graph.

For $\alpha=-\frac{1}{2}$ from (17) $2 R_{-1 / 2} \leq n$ is obtained (see [14, 25]). Closely related with this inequality is the irregularity measure for connected graphs proposed in [19] defined as

$$
\operatorname{Irr}_{R}(G)=1-\frac{2 R_{-1 / 2}}{n} .
$$

Similarly, for $\alpha=-\frac{1}{2}$ and according to (16) the following irregularity measure can be constructed

$$
\operatorname{Irr}_{4}(G)=\sqrt{\frac{4 d_{1}}{n^{2} d_{n}}} R_{-1 / 2}-1 .
$$

Also, for $\alpha=-1$ based on (16) and (17) the following Shi measures can be defined

$$
I r r_{S R 1}=\frac{2 d_{1} R_{-1}}{n}-1
$$

and

$$
\operatorname{Irr}_{S R 2}=1-\frac{2 d_{n} R_{-1}}{n}
$$


Now we mention some irregularity measures for line graphs that can be directly derived from the aforementioned inequalities. Thus, based on (6), (9), (13) and (15) the following irregularity measures for line-graphs can be derived

$$
\begin{gathered}
\operatorname{LIrr}_{1}(G)=\sqrt{\frac{m E M_{1}}{\left(M_{1}-2 m\right)^{2}}-1}, \quad M_{1} \neq 2 m, \\
\operatorname{LIr}_{2}(G)=\sqrt{\frac{2 m^{2} E M_{2}}{\left(M_{1}-2 m\right)^{3}}-1}, \quad M_{1} \neq 2 m, \\
\operatorname{LIrr}_{3}(G)=\frac{\left(M_{1}-2 m\right)^{2 m} E M_{1}}{m^{3}}-1,
\end{gathered}
$$

and

$$
\operatorname{Lrr}_{4}(G)=\frac{\left(M_{1}-2 m\right)^{m} E A}{m^{2}}-1
$$

\section{Acknowledgement}

This research was supported by the Serbian Ministry of Education and Science, under grant TR32012 and TR32009.

\section{References}

[1] H. Abdo, S. BRAndt, D. Dimitrov, The total irregularity of a graph, Discr. Math. Theor. Comput. Sci., 16 (2014), 201-206.

[2] M. O. Albertson, The irregularity of a graph, Ars Comb., 46 (1997), 219-225.

[3] F. K. BELL, A note on the irregularity of a graph, Linear Algebra Appl., 161 (1992), 45-54.

[4] N. BIGGS, Algebraic graph theory, $2^{\text {nd }}$ Edition, Cambridge Univ. Press, Cambridge, 1993.

[5] M. CAVERS, The normalized Laplacian matrix and general Randić index of graphs, A. Thesis, University of Regina, Saskichewan, 2010.

[6] M. CaVers, S. Fallat, S. KiRland, On the normalized Laplacian energy and general Randic index $R_{-1}$ of graphs, Linear Algebra Appl., 433 (2010), 172-190.

[7] F.R. K. Chung, Spectral graph theory, Amer. Math. Soc., Providence, 1997.

[8] L. Collatz, U. Sinogowitz, Spektren endlicher Graphen, Abh. Math. Sem. Univ Hamburg, 21 (1957), 63-77.

[9] K. Ch. Das, K. Xu, J. NAM, Zagreb indices of graphs, Front. Math. China, 10 (3) (2015), $567-582$.

[10] N. DE, Some bounds of reformulated Zagreb indices, Appl. Math. Sci., 6 (101) (2012), 50055012. 
[11] C. S. EDWARDS, The largest vertex degree sum for a triangle in a graph, Bull. London Math. Soc., 9 (1977), 203-208.

[12] C. ElPhick, P. WocJan, New measures of graph irregularity, El. J. Graph. Theory Appl., 2 (1) (2014), 52-65.

[13] G. H. FATH-TABAR, Old and new Zagreb indices of graphs, MATCH Commun. Math. Comput. Chem., 65 (2011), 79-84.

[14] O. Favaron, M. Maheo, J. F. Sacle, Some eigenvalue properties of graphs (Conjectures of Grafitti -II), Discr. Math., 111 (1993), 197-220.

[15] F. GoldBerg, Spectral radius minus average degree: a better bound, arXiv: $1407.4285 \mathrm{v} 1$ [math.co] 16 July, 2014.

[16] I. Gutman, Robbiano, E. Andrade Mortins, D. M. Cordoso, L. Medino, O. RoJo, Energy of line graphs, Linaear Algebra Appl., 433 (2010), 1312-1323.

[17] I. Gutman, B. Furtula (Eds.), Recent results in the Theory of Randic index, Univ. Kragujevac, Kragujevac, 2008.

[18] I. Gutman, B. Furtula (Eds.), Novel molecular structure descriptors, I, Univ. Kragujevac, Kragujevac, 2010.

[19] I. Gutman, B. Furtula, C. ElPhick, The new/old vertex-degree-based topological indices, MATCH Commun. Math. Comput. Chem., 72 (2014), 617-632.

[20] I. Gutman, N. TRinajstić, Graph theory and molecular orbitas. Total $\pi$-electron energy of alternant hydrocarbons, Chem. Phys. Lett., 17 91972), 535-538.

[21] A. HAMZEH, T. RETI, An analogue of Zagreb index inequality obtained from graph irregularity measures, MATCH Commun. Math. Comput. Chem.,72 (2014), 669-683.

[22] J. HAO, Theorems about Zagreb indices and modified Zagreb indices, MATCH Commun. Math. Comput. Chem., 65 (2011), 659-670.

[23] A. Ilić, D. Stevanović, On comparing Zagreb indices, MATCH Commun. Math. Comput. Chem., 62 (2009), 681-687.

[24] A. Ilić, B. Zhou, On reformulated Zagreb indices, Discr. Appl. Math., 160 (2012), 204-209.

[25] X. LI, I. Gutman, Mathematical aspects of Randić -type molecular structure descriptors, Univ. Kragujevac, Kragujevac, 2006.

[26] B. Liu, I. Gutman, Estimating the Zagreb and the general Randic indices, MATCH Commun. Math. Comput. Chem., 57 (2007), 617-632.

[27] M. LiU, B. LiU, On sum of powers of the singless Laplacian eigenvalues of graphs, Hacettepe J. Math. Statis., 41 (4) (2012), 527-536.

[28] A. Milićević, S. Nikolić, On variable Zagreb idices, Croat. Chem. Acta, 77 (2004), $97-$ 101.

[29] A. MilićEviĆ, S. Nikolić, N. TRinajstić, On reformulated Zagreb indices, Mol. Divers., 8 (2004), 393-399.

[30] D. S. Mitrinović, J. E. PeČARIĆ, A. M. FinK, Classical and new inequalities in analysis, Kluwer Academic Publishers, Dordrecht, 1993. 
[31] D. S. Mitrinović, P. M. VAsić, Analytic inequalities, Springer Verlag, Berlin, 1970.

[32] V. NiKIfOROV, Eigenvalues and degree deviation in graphs, Linear Algebra Appl., 414 (2006), 347-360.

[33] S. Nikolić, G. Kovačević, A. Milićević, N. Trinajstić, The Zagreb indices 30 years after, Croat. Chem. Acta, 76 (2003), 113-124.

[34] M. RANDić, On characterization of molecular branching, J. Am. Chem. Soc., 97 (1975), 6609-6615.

[35] L. SHI, Bounds on Randić indices, Discr. Appl. Math., 309 (2009), 5238-5241.

[36] G. Tian, T. HuAng, S. CUI, Bounds on the algebraic connectivity of graphs, Advances Math. 41 (2) (2012), 217-224.

[37] R. Todeschini, V. Consonni, Handbook of molecular descriptors, Wiley-VCH, Weinheim, 2000.

[38] R. Todeschini, V. COnsonni, Molecular descriptors for chemoinformatics, Wiley-VCH, Weinheim, 2009.

[39] N. TRInAjstić, Chemical graph theory, 2nd revised ed., CRC Press, Boca Ration, FL, 1992.

[40] D. Vukičević, I. Gutman, B. Furtula, V. Andova, D. Dimitrov, Some observations on comparing Zagreb indices, MATCH Commun. Math. Comput. Chem., 66 (2011), 627-645.

[41] D. VukičEvić, N. TRINAJstić, Modified Zagreb $M_{2}$ index - comparison with the Randić connectivity index for benzenoid systems, Croat. Chem. Acta, 76 (2003), 183-187.

[42] Y. S. Yoon, J. K. KIM, A relationship between bounds on the sum of squares of degrees of a graph, J. Appl. Math Comput., 21 (2006), 233-238.

[43] B. Zhou, W. LuO, A note on general Randić index, MATCH Commun. Math. Comput. Chem., 62 (2009), 155-162.

[44] P. ZuMSTEIn, Comparison of spectral methods through the adjacency matrix and the Laplacian of a graph, Th. Diploma, ETH Zürich, 2005. 